\title{
A comparison of adaptive management and real options approaches for environmental decisions under uncertainty
}

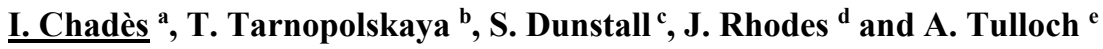 \\ ${ }^{a}$ CSIRO, Dutton Park, QLD \\ ${ }^{b}$ CSIRO, North Ryde, NSW \\ ${ }^{c}$ CSIRO, Clayton, VIC \\ ${ }^{d}$ School of Geography, The University of Queensland, St Lucia, QLD \\ e Fenner School of Environment and Society, The Australian National University, ACT. \\ Email: Iadine.Chades@csiro.au
}

\begin{abstract}
Two approaches to sequential decisions under uncertainty in the environmental management adaptive management and real options analysis - have evolved independently over the last decades.

Adaptive management, or learning by doing, originated from adaptive control. Adaptive management is acknowledged as one of the best-practice methods to manage biological systems under structural uncertainty. Adaptive management has been used for the management of renewable natural resources (such as fisheries and waterfowl) and the conservation of species (such as assisted colonization, restoration and threatened species management). In this context, stochastic dynamic models and historical data would be valuable for describing and predicting responses of management decisions, but these are either non-existent or severely limited in their scope.
\end{abstract}

Real options analysis originated from mathematical finance and is based on financial options pricing theory. The real options analysis can be viewed as both sequential decision-making and project valuation in a highly uncertain environment with non-stationary dynamics. Real options analysis has most often been used for industrial applications (such as mining, asset management, infrastructure, energy, defence, and agriculture). In this context, reasonably good stochastic dynamic models and historical data exist for describing and forecasting the behaviour of risk factors.

In mathematical terms, both adaptive management and real options approaches are based on stochastic optimal control and Markov decision processes. In environmental decision-making both enable practitioners and managers to make optimal decisions under uncertainty. However, the numerical methods of solving adaptive management versus real options problems are different, as their development has been motivated by the different needs of respective application areas. An important feature of adaptive management is the presence of and need to account for a small number of hidden variables. In contrast, real options focus on the development of techniques capable of dealing with high-dimensional problems with multiple stochastic risk factors.

Limited for a long time by the inefficiency of the solution methods, recent advances in both adaptive management and real options now allow us to solve more realistic environmental decision problems under uncertainty, widening the scope of their applications. Growing availability of data in the environmental management arena and an emerging need to conduct industrial operations in the proximity of conservation areas will require new decision-making approaches that can combine recent advances in adaptive management and real options.

This paper reviews recent advances in both adaptive management and real options methodologies, and compares methods for solving decisions under uncertainty problems based on the type of uncertainty they are addressing, the type of decision-making approach, important assumptions, and the size of the problems they are capable of dealing with. This paper proposes new areas of development that could inspire future research and better-informed environmental decisions under uncertainty.

Keywords: Sequential decisions under uncertainty, stochastic optimal control, environmental decisions 


\section{INTRODUCTION}

Decisions about managing environmental resources efficiently and effectively are complicated by uncertainty. The literature on environmental decisions refers to four kinds of uncertainty: (i) environmental variation or process uncertainty, (ii) partial controllability or stochasticity, (iii) state uncertainty or partial observability and (iv) structural uncertainty (Williams et al., 1996). Despite a range of approaches available to deal with uncertainty in environmental decisions, no approaches are available to solve large-size problems under all uncertainties. Selecting a particular approach to decision-making implies a trade-off in dealing better with some types of uncertainty over others.

Adaptive management and real options approaches for sequential decisions under uncertainty in environmental management have undergone significant evolution over the last two decades. Both approaches are based on stochastic optimal control and Markov decision processes, they evolved independently from each other and their developments were motivated by different needs. Adaptive management was specifically developed to handle decision problems with imperfect knowledge of the dynamics of the system, and is known as 'learning by doing'. On the other hand, real options analysis was introduced specifically to value the flexibility to change actions over time in response to the evolution of uncertainty, and represents both optimal sequential decisions under uncertainty and a capital budgeting methodology. Because of these different purposes, different analytic and numerical methods were developed to solve these problems.

Recent advances in both adaptive management and real options now allow us to solve more realistic environmental decision problems under uncertainty, widening the scope of their applications. Growing availability of data in the environmental management arena and an emerging need to conduct industrial operations in the proximity of conservation areas require new decision-making approaches that could make use of the combined recent advances in adaptive management and real options.

This paper reviews and compares the concepts, applications and recent advances in the numerical and analytic techniques in adaptive management and real options methodologies. A large body of knowledge accumulated in both fields makes a comprehensive review impractical in the context of this paper. Therefore, our review focuses on the most recent developments, with the purpose to identify potential areas of new developments that would address new challenges in the environmental decision area. The paper is organized as follows. Section 2 and 3 review the concepts, applications in environmental decisions, and new advanced methods for adaptive management and real options respectively. Section 4 compares two approaches, and, finally, potential new directions are briefly discussed in Section 5.

\section{ADAPTIVE MANAGEMENT}

Adaptive management, or learning by doing, is acknowledged as the best practice method to manage biological systems under uncertainty. Mathematically, an adaptive management problem can be formulated as a sequential decision problem. An optimal solution provides the best suite of actions to implement so that the chance of achieving an objective over time is maximized. Solving adaptive management problems requires applying three mathematical concepts. Markov decision processes (MDP) are used to find optimal sequential decisions. Sufficient statistics compactly represent current knowledge on uncertain quantities based on past information. Bayes' theorem allows us to predict and reduce the uncertainty by updating sufficient statistics.

Adaptive management was specifically developed to handle decision problems with structural uncertainty that corresponds to imperfect knowledge of the system dynamics. Two kinds of structural uncertainty are considered in the adaptive management literature: parameter uncertainty and model uncertainty. Parameter uncertainty assumes that the model of the dynamics is known but the parameters of the model dynamics are unknown. For example, population dynamics may be defined as a Beverton-Holt population model with an unknown growth parameter or carrying capacity. Model uncertainty assumes we do not know the model dynamics of the system. For example, it might be unclear whether or not a population dynamics follows a Beverton-Holt or Ricker type model. The available model dynamics are often limited to a small number of alternatives (discrete set). To simplify these concepts, it is useful to realize that in both cases, parameter or model uncertainty, the optimization problem is solving a sequential decision problem under uncertainty with hidden variables (Chadès et al., 2012). These hidden variables might be continuous (parameter uncertainty) or discrete (parameter or model uncertainty).

Once the type of structural uncertainty is identified, two solution methods are available: active and passive adaptive management. Active adaptive management provides the best action to implement assuming that the current knowledge of the system might not be correct, predicting the mistakes that may arise and future 
improvement as management proceeds over time. Solutions to active adaptive management problems maximize the chance of achieving the objective by explicitly accounting for future learning opportunities. In contrast, passive adaptive management does not explicitly account for future learning opportunities. From an optimization perspective, passive adaptive management methods are easier to solve and provide an approximation to the active adaptive management problems. Both approaches are used in the literature.

\subsection{Adaptive management in environmental management}

Under parameter uncertainty, the task is to manage the system while simultaneously learning the value of the parameter to improve future management decisions. Walters and Hilborn (1976) were the first to take advantage of conjugate distributions describing the prior and posterior to streamline Bayesian updating of uncertainty. They modelled the uncertain parameter with a Normal distribution. The advantage of using conjugate distributions is that it is possible to obtain a closed-form expression for the posterior, so the distribution can be updated exactly and without resorting to numerical simulation methods. For example, McCarthy and Possingham (2007) posed a problem where a manager must choose between implementing two actions, both with unknown probabilities of success. Each probability of success is described by a Beta distribution with parameters $\alpha$ and $\beta$. These sufficient statistics are updated after observing s successes and $\mathrm{f}$ failures from the trials implemented, as $\alpha+\mathrm{s}$ and $\beta+\mathrm{f}$. This relationship between a probability described by a Beta distribution and binomial (success/failure) updating process forms the basis of most recent work on adaptive management for parameter uncertainty (Hauser and Possingham, 2008; Rout et al., 2009).

First used in the fisheries literature (Silvert, 1978; Walters, 1986), model uncertainty is represented as alternative models of the system dynamics. Models can take many forms, so long as transition probabilities between states can be computed. Adaptive management under model uncertainty was implemented by the US Fish and Wildlife Service to set harvest quotas for mallards in the USA (Nichols et al., 1995; Johnson et al., 1997), which set the stage for other adaptive management studies designed to reduce model uncertainty in conservation and resource management (Moore and Conroy, 2006; Martin et al., 2009; McDonald-Madden et al., 2010). Perhaps because the solution methods for reducing model uncertainty are more general than those designed to reduce parameter uncertainty, the majority of adaptive management studies rely on methods for reducing model uncertainty rather than parameter uncertainty.

\subsection{New advanced methods using POMDP}

Adaptive management was first called adaptive control in reference to the control theory literature from which it first originated (Walters and Hilborn, 1976). Since Walter's seminal book (Walters, 1986), adaptive management grew independently from the optimisation literature and advances in solving sequential decision problems did not reach the adaptive management literature until recently. As a result, adaptive management was only ever really applied to problems with less than 1,000 states and a maximum of 3 uncertain alternative models. Perhaps a turning point to solve adaptive management under model uncertainty was the work by (Williams, 2011), where the author concluded that adaptive management and partially observable decision processes (POMDP) shared some similarities but these two approaches were different. Chadès et al. (2012) demonstrated that an adaptive management problem can be modelled as a simpler POMDP and solved using the repertoire of fast POMDP solution methods from artificial intelligence. These fast solution methods are capable of solving problems with hundreds of thousands of states, including point based approaches as demonstrated in (Nicol et al., 2013). A POMDP is an augmented MDP that accounts for the influence of hidden variables in the decision process. Using POMDP, Nicol et al. (2015) solved an ambitious active adaptive management problem assuming non-stationary dynamics of migratory shorebird populations threatened by habitat loss due to sea level rise (800,000 states, 3 models, 8 actions).

Under parameter uncertainty, it is not always possible to find an exact conjugate prior for every parameter uncertainty problem and numerical solutions may be required to update sufficient statistics. Density projections for distributions from the exponential family can provide an alternative by calculating the posterior distribution by projecting the continuous belief space of the unknown parameter to the closest discrete distribution that matches the family of the prior distribution. This projected belief becomes a continuous-state MDP which can be solved in a number of ways. In resource management, this has been applied to a hierarchical beta distributed model with both continuous action and belief state spaces on a small size problem (Springborn and Sanchirico, 2013). 


\section{REAL OPTIONS ANALYSIS}

A key concept behind real options analysis is that uncertainty and the company's ability to adapt to it (flexibility) can create value-adding opportunities. Real options analysis extends financial options pricing theory to options on real assets. The financial call option gives the investor the right (but not the obligation) to purchase an asset should its value rise above a specific threshold (strike price). By analogy, the 'real option' gives the right (but not the obligation) to undertake a certain business activity at some time in the future. Thus, a real options approach makes it possible to capitalise on desirable developments, whilst protecting against undesirable future developments. The purpose of real options analysis is to compute the value of flexibility generated by options.

Real options valuation is based on financial contingent claim analysis, where the option value is the discounted expected value of the future payoffs under the unique risk-neutral measure, which exists only in the arbitrage-free market (i.e. when there are no opportunities to make money given no initial investment without a possibility of loss). Real options analysis is usually based on American options pricing, which is an optimal stopping problem in the control field. Inherent in real options analysis is an optimisation of timing for actions under uncertainty. The real options analysis can be viewed as both sequential decision-making and capital budgeting (dynamic project valuation) in a highly uncertain environment.

\subsection{Real options analysis in environmental management}

Real options analysis has generated a considerable and a growing interest in the fields of industrial and environmental decisions over the last two decades (Dixit and Pindyck, 1994). A thorough review of environmental applications is given by Mezey and Conrad (2010). Early applications of real options in conservation planning focused on the amount of wilderness to develop when the future value of wilderness was stochastic (Arrow and Fisher (1974); and on the spatial allocation of natural resources between two competing and mutually exclusive uses: conservation and exploitation. Recent examples include valuing: (a) the flexibility associated with species substitutability, as a source of biodiversity value (Kassar and Lasserre, 2004); (b) a decision to stop timber harvesting when a woodland caribou population becomes threatened with extinction (Morgan et al., 2007); (c) a decision to convert an area of land used as a biodiversity reserve to an alternative use, such as agricultural production (Leroux et al., 2009); (d) individual rehabilitation projects, accounting for the effects of climate change on species loss, future rehabilitation benefits and frequency of catastrophic events (Leroux and Whitten, 2014).

Applications of real options in environmental management typically consider a single real option under a single stochastic risk factor. The stochastic processes assumed (e.g., the dynamics of recovered ecological value from rehabilitation, species values, temporal variability of caribou habitat, etc.) do not require advanced numerical techniques (in many cases, analytic solutions are possible). Mezey and Conrad (2010) suggest that the application of real options analysis for conservation planning and for adapting to climate change will be important areas for future research, and that this would require a broader range of stochastic processes and new techniques for solving valuation and management problems.

\subsection{New advanced methods for high-dimensional problems}

Early applications of real options analysis studied optimal timing to implement a single real option under one or two stochastic risk factors. Techniques used to solve such a problem included stochastic dynamic programming, binomial and trinomial lattices, and analytic and numerical solutions of stochastic partial differential equations. All these techniques suffer from the 'curse of dimensionality' and are not suitable for high-dimensional problems.

In the last decade, there emerged a recognition that realistic real options problems in resource management and other industries often involve multiple interacting options (decisions) as well as multiple stochastic risk factors (state variables). Mathematically, such problems can be viewed as optimal stochastic switching problems. New advanced methods are required to solve such problems in realistic dimensions. These new approaches to analysing complex real options problems can be roughly divided into the two main categories:

1. Stochastic mixed-integer programming approach. This approach is proposed and discussed in details in Brosch (2008). This approach can accommodate a large number of constraints, however due to the curse of dimensionality, the maximum number of risk factors considered so far is three.

2. Regression Monte Carlo approach. This approach is based on value function approximation via basis function, and was popularised by Longstaff and Schwartz (2001)) under the name Least-Squares Monte Carlo (LSMC). 
LSMC is a very versatile simulation-based technique that is easily extendable to higher dimensions. The LSMC approach consists of two parts: (1) in the forward loop, a sample of all the risk factors is simulated forward in time, using Monte Carlo simulations; (2) in the backward loop, the Bellman's optimality principle is used to compute the optimal control on each sample path, using regression estimates for the continuation values, based on cross-sectional information. However, LSMC has limitations that make its extension to higher dimensions problematic: (1) difficulties in choosing an appropriate regression basis and unclear dependence between the Monte Carlo sample size and the regression basis dimension required for convergence; (2) possibility of 'over-fitting' when polynomial basis functions are used; and (2) memory complexity. LSMC has been extended to more general regression methods, known as regression Monte Carlo, and more general stochastic control problems, such as optimal switching problems. A comprehensive review can be found in Bouchard and Warin (2012). Most promising recent improvements include: control randomization, memory reduction and fast adaptive local regression (Langrene et al., 2015), (Aïd et al., 2013). One of the advantages of regression Monte Carlo approach is that a synthesis of optimal control can be constructed (see, e.g., Tarnopolskaya et al., 2015). The algorithm that combines dynamic programming, Monte Carlo simulations, adaptive local basis regression and memory reduction techniques can solve problems with large number of actions, up to 10 stochastic risk factors and long time horizons (Aïd et al., 2013).

\section{A COMPARISON OF ADAPTIVE MANAGEMENT AND REAL OPTIONS ANALYSIS}

Sections 2 and 3 show that both adaptive management and real options approaches are methodologies for optimal sequential decisions under uncertainty and are based on stochastic optimal control and Markov decision processes as an underlying mathematical methodology. However, there are considerable differences in their concepts, attitude to uncertainty, objectives, and numerical and analytic techniques. The differences are summarized in Table 1.

Interestingly, in environmental management and in particular conservation, uncertainty is perceived to make decisions more difficult and may hinder decision-making despite the urgency of the situation and available methods such as adaptive management to reduce uncertainty (Martin et al., 2012). In comparison, in real options analysis uncertainty is "harnessed" to provide added benefits to an otherwise status quo management decision. The uncertainty in the system and decisions available are synonymous with opportunities to find better choices, also coined as "flexibility". Such a difference in the attitude towards uncertainty could be partially explained by another difference between the two approaches: real options analysis is often related to maximizing a monetary value, while adaptive management problems often deal with biological systems for which monetary values are not always available or when available, subject to heated debate.

Distinctive features of adaptive management come from the lack of readily accessible data to make future predictions and build reliable models. Adaptive management focuses on learning about the system through management by accounting for structural uncertainty and hidden variables. Perhaps as a consequence of few data, a drawback of existing adaptive management methods is the inability to deal with multiple stochastic risk factors (or state variables). Additionally, to increase the chance of uptake, solutions must be expressed as

Table 1. Comparison of Adaptive Management and Real Options Approaches

\begin{tabular}{lll}
\hline & Adaptive Management & Real Options \\
\hline $\begin{array}{l}\text { Attitude to } \\
\text { uncertainty }\end{array}$ & $\begin{array}{l}\text { Reduce structural uncertainty to maximise } \\
\text { management outcomes. }\end{array}$ & $\begin{array}{l}\text { Harnessing uncertainty to add value though optimal } \\
\text { management. }\end{array}$ \\
\hline $\begin{array}{l}\text { Types of } \\
\text { uncertainty }\end{array}$ & $\begin{array}{l}\text { Process uncertainty, structural uncertainty (model and } \\
\text { parameter uncertainties) and partial observability. }\end{array}$ & Process uncertainty, parameter uncertainty \\
\hline $\begin{array}{l}\text { Mathematical } \\
\text { model }\end{array}$ & $\begin{array}{l}\text { Markov property assumed, modelled as a MDP or } \\
\text { POMDP with state space augmented with sufficient } \\
\text { statistics. Probability transitions from state-action to } \\
\text { state pre-calculated (not simulated). }\end{array}$ & $\begin{array}{l}\text { Markov property assumed, modelled as optimal stopping } \\
\text { and optimal stochastic switching (MDP). Solved using } \\
\text { regression Monte Carlo approach (a combination of DP, } \\
\text { Monte Carlo simulations and value function approximation) }\end{array}$ \\
\hline $\begin{array}{l}\text { Role of } \\
\text { learning }\end{array}$ & $\begin{array}{l}\text { Learning is part of the decision process } \\
\text { Purpose }\end{array}$ & $\begin{array}{l}\text { Real options analysis share similarities with passive } \\
\text { adaptive management }\end{array}$ \\
\hline $\begin{array}{l}\text { Features of } \\
\text { advanced } \\
\text { methods }\end{array}$ & $\begin{array}{l}\text { Small number of risk factors; non stationary } \\
\text { dynamics; large states space; small action space; } \\
\text { imperfect detection. }\end{array}$ & $\begin{array}{l}\text { Capital budgeting and optimal flexible management } \\
\text { large state and action space }\end{array}$ \\
\hline Problem size & $\begin{array}{l}\text { The largest problem solved has 800,000 discrete } \\
\text { states, less than 10 actions, 1 hidden stochastic risk } \\
\text { factor and infinite time horizon (Nicol et al., 2015) }\end{array}$ & $\begin{array}{l}\text { Continuous state space sampled via Monte Carlo } \\
\text { simulations (up to 1,000,000 realizations), up to 50 actions; } \\
\text { up to 10 stochastic risk factors; long time horizons }\end{array}$ \\
\hline
\end{tabular}


simply as possible - a challenge when dealing with hidden variables. Methods that trade off simplicity and optimality have recently been developed to facilitate uptake of POMDP solutions (Dujardin et al., 2015). Real options analysis assumes the parameters of the stochastic processes are known, or their relative values can be assessed.

The important distinctive features of real options analysis are its focus on valuing flexibility. New numerical techniques originated from financial options pricing capable of solving problems with multiple stochastic risk factors (to the authors' knowledge, no other methods are suitable for solving high-dimensional stochastic control problems). In addition, new regression Monte Carlo techniques for real options analysis are flexible and easily accommodate different types of stochastic process dynamics including non-stationary dynamics. However, real options analysis does not explicitly account for future learning opportunities and, in this sense, shares some similarities with passive adaptive management (Sanderson et al., 2015).

\section{FUTURE DIRECTIONS}

Today the volume, variety and velocity of data on environmental and human systems are increasing. Furthermore, growing human populations and development needs demand trading-off biodiversity needs. New decision-making approaches are urgently required that are capable of dealing with multiple uncertainties, including increased data loads from multiple sources that vary in quality, working in an environment of uncertain future change, and an imperfect understanding of system dynamics and responses to managerial actions.

Combining advances from the adaptive management and the real options areas may provide a way forward. First, adaptive management may benefit from incorporating valuation of flexibility into the modelling framework. Such an extension would be especially suitable for passive adaptive management. The current attitude towards uncertainty in adaptive management literature is that it adds complexity rather than adding value (a risk-averse approach). Systematically demonstrating the value of adapting to uncertainty by accepting risk could improve the impact of adaptive management. Second, in real options the choice of the system dynamics model significantly affects the options value. Improving our understanding of system dynamics by combining adaptive management techniques of reducing model uncertainty with real options would present a significant advance. From a real options viewpoint, adaptive management reveals the value of information and active learning could be included in real options. Third, the flexibility of regression Monte Carlo methods presents an opportunity to extend them to incorporate model uncertainty and learning (feedback) into a single approach, in order to develop new numerical techniques for adaptive management that are capable of dealing with multiple stochastic risk factors. Finally, if the two approaches were to have a stronger impact to environmental decision problems, accounting for multi-objectives in both adaptive management and real options would provide opportunities to reconcile diverging point of views towards uncertainty.

\section{REFERENCES}

Aïd, R., L. Campi, N. Langrené, and H. Pham (2013). A probabilistic numerical method for optimal multiple switching problems in high dimension. SIAM Journal of Financial Mathematics, 5(1), 191-231.

Arrow, K.J., and A.C. Fisher (1974). Environmental preservation, uncertainty, and irreversibility. The Quarterly Journal of Economics, 88(2), 312-319.

Bouchard, B., and X. Warin (2012). Monte-Carlo valuation of American options: facts and new algorithms to improve existing methods. In: Numerical Methods in Finance (eds. Carmona, R, P Del Moral, H Peng, and N Oudjane). Springer New York, pp. 215-255.

Brosch, R. (2008). Portfolios of Real Options, Springer-Verlag, Berlin.

Chadès, I., J. Carwardine, T.G. Martin, S. Nicol, R. Sabbadin, and O. Buffet (2012). MOMDPs: a solution for modelling adaptive management problems. The Twenty-Sixth AAAI Conference on Artificial Intelligence (AAAI-12), pp. 267-273, Toronto, Canada.

Dixit, A.K., and R.S. Pindyck (1994). Investment Under Uncertainty, Princeton University Press, Princeton.

Dujardin, Y., T. Dietterich, and I. Chades (2015). alpha-min: A Compact Approximate Solver for FiniteHorizon POMDPs. International Joint Conference on Artificial Intelligence, IJCAI-2015, p. 8, Buenos Aires.

Hauser, C.E., and H.P. Possingham (2008). Experimental or precautionary? Adaptive management over a range of time horizons. Journal of Applied Ecology, 45(1), 72-81.

Johnson, F.A., T.M. Clinton, W.L. Kendall, J.A. Dubovsky, D.F. Caithamer, J.R. Kelley, Jr., and K.W. Byron (1997). Uncertainty and the management of mallard harvests. The Journal of Wildlife Management, 61(1), 202-216. 
Kassar, I., and P. Lasserre (2004). Species preservation and biodiversity value: a real options approach. Journal of Environmental Economics and Management, 48(2), 857-879.

Langrene, N., T. Tarnopolskaya, W. Chen, Z. Zhu, and M. Cooksey (2015). New regression Monte Carlo methods for high-dimensional real options problems in minerals industry. 22nd International Congress on Modelling and Simulations (MODSIM), p. 8, Gold Coast.

Leroux, A.D., V.L. Martin, and T. Goeschl (2009). Optimal conservation, extinction debt, and the augmented quasi-option value. Journal of Environmental Economics and Management, 58(1), 43-57.

Leroux, A.D., and S.M. Whitten (2014). Optimal investment in ecological rehabilitation under climate change. Ecological Economics, 107, 133-144.

Longstaff, F.A., and E.S. Schwartz (2001). Valuing American options by simulation: a simple least-squares approach. Review of Financial studies, 14(1), 113-147.

Martin, J., M.C. Runge, J.D. Nichols, B.C. Lubow, and W.L. Kendall (2009). Structured decision making as a conceptual framework to identify thresholds for conservation and management. Ecological Applications, 19(5), 1079-1090.

Martin, T.G., S. Nally, A.A. Burbidge, S. Arnall, S.T. Garnett, M.W. Hayward, L.F. Lumsden, P. Menkhorst, E. McDonald-Madden, and H.P. Possingham (2012). Acting fast helps avoid extinction. Conservation Letters, 5(4), 274-280.

McCarthy, M.A., and H.P. Possingham (2007). Active adaptive management for conservation. Conservation Biology, 21(4), 956-963.

McDonald-Madden, E., W.J.M. Probert, C.E. Hauser, M.C. Runge, H.P. Possingham, M.E. Jones, J.L. Moore, T.M. Rout, P.A. Vesk, and B.A. Wintle (2010). Active adaptive conservation of threatened species in the face of uncertainty. Ecological Applications, 20(5), 1476-1489.

Mezey, E.W., and J.M. Conrad (2010). Real options in resource economics. Annual Review of Resource Economics, 2(1), 33-52.

Moore, C.T., and M.J. Conroy (2006). Optimal regeneration planning for old-growth forest: addressing scientific uncertainty in endangered species recovery through adaptive management. Forest Science, 52(2), 155-172.

Morgan, D., S.B. Abdallah, and P. Lasserre (2007). A real options approach to forest-management decision making to protect caribou under the threat of extinction. Ecology and Society, 13(1), 27.

Nichols, J.D., F.A. Johnson, and K.W. Byron (1995). Managing north american waterfowl in the face of uncertainty. Annual Review of Ecology and Systematics, 26, 177-199.

Nicol, S., O. Buffet, T. Iwamura, and I. Chades (2013). Adaptive management of migratory birds under sea level rise. Proceedings of the 23rd International Joint Conference on Artificial Intelligence, pp. 2955-2957, Beijing.

Nicol, S., R.A. Fuller, T. Iwamura, and I. Chadès (2015). Adapting environmental management to uncertain but inevitable change. Proceedings of the Royal Society B, 282(1808).

Rout, T.M., C.E. Hauser, and H.P. Possingham (2009). Optimal adaptive management for the translocation of a threatened species. Ecological Applications, 19(2), 515-526.

Sanderson, T., G. Hertzler, T. Capon, and P. Hayman (2015). A real options analysis of Australian wheat production under climate change. Australian Journal of Agricultural and Resource Economics, doi: 10.1111/1467-8489.12104.

Silvert, W. (1978). The price of knowledge: Fisheries management as a research tool. Journal of the Fisheries Research Board of Canada, 35(2), 208-212.

Springborn, M., and J.N. Sanchirico (2013). A density projection approach for non-trivial information dynamics: adaptive management of stochastic natural resources. Journal of Environmental Economics and Management, 66(3), 609-624.

Tarnopolskaya, T., W. Chen, and C. Bao (2015). Switching boundaries for flexible management of natural resource investment under uncertainty. IAENG Transactions on Engineering Sciences, 1-14.

Walters, C.J. (1986). Adaptive management of renewable resources, McGraw Hill, New York, NY.

Walters, C.J., and R. Hilborn (1976). Adaptive control of fishing systems. Journal of the Fisheries Research Board of Canada, 33(1), 145-159.

Williams, B.K. (2011). Resolving structural uncertainty in natural resources management using POMDP approaches. Ecological Modelling, 222(5), 1092-1102.

Williams, B.K., F.A. Johnson, and K. Wilkins (1996). Uncertainty and the adaptive management of waterfowl harvests. Journal of Wildlife Management, 60(2), 223-232. 